\title{
Twofold-broken rational tori and the uniform semiclassical approximation
}

\author{
H. Schomerus ${ }^{\mathrm{a}}$ \\ Instituut-Lorentz, Universiteit Leiden, P.O. Box 9506, 2300 RA Leiden, The Netherlands
}

Received 3 August 1999

\begin{abstract}
We investigate broken rational tori consisting of a chain of four (rather than two) periodic orbits. The normal form that describes this configuration is identified and used to construct a uniform semiclassical approximation, which can be utilized to improve trace formulae. An accuracy gain can be achieved even for the situation when two of the four orbits are ghosts. This is illustrated for a model system, the kicked top.
\end{abstract}

PACS. 05.45.Mt Semiclassical chaos ("quantum chaos") - 03.65.Sq Semiclassical theories and applications

\section{Introduction}

Periodic orbits provide the skeleton of the dynamics of classical Hamiltonian systems. Generic dynamical systems display a mixed phase space, consisting of islands of stability residing in chaotic seas. The periodic orbits are neither grouped in families, like in integrable systems, nor are they well isolated and all unstable, as for chaotic systems. One rather finds also stable periodic orbits surrounded by islands of regular behaviour. These islands of stability look locally like an almost integrable system, altogether with a KAM structure of invariant tori and chains of periodic orbits, remnants of rational tori of a supposedly contiguous integrable situation [1].

In a semiclassical treatment of the corresponding quantum system, clusters of proximate orbits display a collective behaviour. The bifurcations at the centre of the island and their semiclassical treatment have been addressed in a number of recent works, both for the generic variants $[2-6]$ as well as for classically non-generic, but semiclassically still relevant cases $[7,8]$.

In [9] the class of near-integrable systems has been addressed, and a uniform semiclassical approximation for the most frequently encountered broken rational tori (consisting of a stable and an unstable periodic orbit) was presented. We will call these tori the "simple" tori. Interestingly, the semiclassical approximation works reasonably well even beyond the point where the stable orbit becomes unstable [10]. The same configuration of a stable and an unstable orbit is also typical close (in parameter space) to most types of period- $n$-tupling bifurcations at the centre of a stability island. For bifurcation number $n \geq 5$, for the island-chain scenario with $n=4$, and also (as a consequence of a more complicated bifurcation scenario) for $n=3$, two satellite orbits are expelled from the centre. At

\footnotetext{
a e-mail: henning@lorentz.leidenuniv.nl
}

a certain distance from the bifurcation the satellite orbits can be treated as isolated from the central orbit; however, a collective semiclassical treatment of the two satellites is often still necessary, and this can be achieved by using the abovementioned approximation for the simple torus.

Although encountered less frequently, there are situations where a broken torus consists not of two, but a higher number of periodic orbits. In the islands of stability tori of this type appear especially at larger distance from a bifurcation. In this work we study the twofold-broken rational torus, consisting of two stable and two unstable periodic orbits. It is described by a normal form which is obtained from the normal form of the simple torus by including the second harmonic in an angular coordinate. From the normal form we construct a uniform approximation that can be used to improve semiclassical trace formulae. Indeed, the relevance of this configuration is much enhanced in the semiclassical context: here one has to consider also "ghost" orbits with complex coordinates [11], and even a simple broken torus can be affected by nearby ghosts, making a treatment as a "pre-formed" twofold-broken torus advisable. This is illustrated in a model system, a periodically driven angular momentum vector (the kicked top), where we find a configuration of four period-three orbits which can be regarded as a twofold-broken rational torus. A reduction of the error of the trace formula by a factor of about $2-3$ is found even when two of these satellites are ghosts. An even higher accuracy gain is attained for socalled "inverse- $\hbar$ spectroscopy".

\section{Normal forms and uniform approximations}

We restrict the analysis to two-dimensional areapreserving maps. (The results are also applicable to autonomous Hamiltonian systems with two degrees of freedom.) The quantum version of the map is generated 
by the unitary Floquet operator $F$ which acts on the vectors of a Hilbert space, mapping the space onto itself. The semiclassical trace formula relates the traces $\operatorname{tr} F^{n}$ to the classical periodic orbits. Isolated orbits of primitive period $n_{0}$ give an additive contribution [12-15]

$$
C=A \exp \left[\mathrm{i} \frac{S}{\hbar}-\mathrm{i} \frac{\pi}{2} \mu\right]
$$

with amplitude

$$
A=n_{0}|2-\operatorname{tr} M|^{-1 / 2}
$$

to all traces $\operatorname{tr} F^{n}$ with $n=n_{0} r$ and integer repetition number $r$. Besides the primitive period, three classical quantities of the ( $r$ th return of the) periodic orbit enter, the action $S$, the trace of the linearized $n$-step map $M$, and the Maslov index $\mu$.

The expression (1) is derived by a stationary-phase approximation and becomes inaccurate when orbits lie close together. Then a collective treatment of the region $\Omega$ inhabited by the proximate orbits becomes necessary. This can be achieved by introducing normal forms for a phase function $\Phi$ and an amplitude function $\Psi$ into the more general expression [2-4]

$$
C_{\Omega}=\frac{1}{2 \pi \hbar} \int_{\Omega} \mathrm{d} \varphi^{\prime} \mathrm{d} I \Psi\left(\varphi^{\prime}, I\right) \exp \left[\frac{\mathrm{i}}{\hbar} \Phi\left(\varphi^{\prime}, I\right)-\mathrm{i} \frac{\pi}{2} \nu\right] .
$$

Here $I, \varphi$ are canonical polar (or cylinder) coordinates, and $\nu$ is the Morse index.

The famous Poincaré-Birkhoff theorem states that a perturbation of an integrable system causes tori with rational winding number to break into chains of alternating stable and unstable periodic points. However, it does not give a quantitative criterion for the number of distinct orbits that lie on this chain. The simple broken torus is described by the normal form

$$
S\left(I, \varphi^{\prime}\right)=S_{0}+I \varphi^{\prime}-a I^{2}-b \cos \varphi^{\prime}
$$

for the generating function $S$, with constants $S_{0}, a$, and $b$. The corresponding map $(I, \varphi) \rightarrow\left(I^{\prime}, \varphi^{\prime}\right)$, implicitly given by

$$
\varphi=\frac{\partial S}{\partial I}, \quad I^{\prime}=\frac{\partial S}{\partial \varphi^{\prime}}
$$

is the well-known standard map. This normal form has been used in [9] to obtain a uniform semiclassical approximation for the simple broken torus, smoothly interpolating between the two non-commuting classical and integrable limits ( $\hbar \rightarrow 0$ and $b \rightarrow 0$, respectively).

A more complete picture can be obtained when one includes the second harmonic in the angular variable $\varphi^{\prime}$ and works with the extended normal form

$$
S\left(I, \varphi^{\prime}\right)=S_{0}+I \varphi^{\prime}-a I^{2}-b \cos \varphi^{\prime}-c \cos \left(2 \varphi^{\prime}+2 \varphi_{0}\right) .
$$

The strategy of including higher-order terms in normal forms has been pursued before, with two different incentives. Firstly, the inclusion of higher orders is a tool to equip a normal form with a sufficient number of independent parameters. This can be necessary to account for all classical properties (stabilities and actions) of the periodic orbits described by the normal form. Secondly, the higher orders describe additional periodic orbits, and hence more complicated configurations than the usual normal forms [7,8,16-18]. A semiclassical description often succeeds only when all orbits of an extended normal form are treated collectively. Presently we aim at the inclusion of additional periodic orbits.

The periodic orbits satisfy the fixed point conditions

$$
\begin{aligned}
& I=\frac{\partial S}{\partial \varphi^{\prime}}=I+b \sin \varphi^{\prime}+2 c \sin \left(2 \varphi^{\prime}+2 \varphi_{0}\right) \\
& \varphi^{\prime}=\frac{\partial S}{\partial I}=\varphi^{\prime}-2 a I
\end{aligned}
$$

resulting in $I=0$ and

$$
b \sin \varphi^{\prime}+2 c \sin \left(2 \varphi^{\prime}+2 \varphi_{0}\right)=0 .
$$

This condition amounts to finding the roots of a fourthorder polynomial in $\sin \varphi^{\prime}$. Depending on the parameters $b, c$, and $\varphi_{0}$ there are either four real solutions or two real and two complex solutions. For $|b|<2|c|$ there are always four real solutions. For $|b|>|c|$ only two real solutions are found. When $|b / c|$ is fixed in the range $(1,2)$ and $\varphi_{0}$ is varied one finds tangent bifurcations with two real solutions on one side of the bifurcation and four real solutions on the other side. The real solutions correspond to conventional periodic orbits while the complex solutions are "ghosts". They are of no consequence for the classical dynamics but can be important for the semiclassical description of the quantum system, as has been shown in [11] and as we shall see once more below.

The joint contribution of the orbits on the twofoldbroken torus is found by introducing into equation (3) the normal form

$$
\Phi=S_{0}-a I^{2}-b \cos \varphi^{\prime}-c \cos \left(2 \varphi^{\prime}+2 \varphi_{0}\right)
$$

(cf. Eq. (6)) for the phase function and

$$
\Psi=1+d \cos \left(\varphi^{\prime}+\varphi_{1}\right)+e \cos \left(2 \varphi^{\prime}+2 \varphi_{0}\right)
$$

for the amplitude function. The stationary-phase limit of equation (3) is a sum of four additive contributions of form (1), each representing one orbit. The four parameters $S_{0}, b, c, \varphi_{0}$ are determined by matching the phases of each contribution to the actions $S$ of the periodic orbits. The parameters $a, d, e$, and $\varphi_{1}$ are fixed by the stability amplitudes $A$. This strategy works also when two of the orbits are ghosts: phases and amplitudes become then complex, but are related by complex conjugation, and the number of real independent parameters remains unchanged.

Equations $(3,10,11)$ represent a uniform approximation of the joint contribution of the twofold-broken torus. The integration over $I$ is readily carried out, which leaves 
us with a one-dimensional strongly oscillating integral over the coordinate $\varphi^{\prime}$. Numerically it is most conveniently evaluated by the method of steepest descent, for which the integration contour is deformed into the complex plane. On the new contour the integrand decreases exponentially. The new contour can also visit stationary points with complex coordinates, i.e., ghost orbits.

\section{Numerical results}

We shall illustrate our findings, and especially the relevance of ghosts, for a situation encountered in a model system, the dynamics of a periodically driven angular momentum vector $\mathbf{J}$ (the kicked top [19]). The components of $\mathbf{J}$ obey the usual commutation rules $\left[J_{x}, J_{y}\right]=\mathrm{i} J_{z}$ (and cyclic permutations). The total angular momentum $\mathbf{J}^{2}=j(j+1)$ is conserved, restricting the dynamics to the irreducible representations of the angular-momentum algebra. The Hilbert space dimension is $2 j+1$. The effective Planck's constant is $1 /(j+1 / 2)$ and the classical limit is attained for $j \rightarrow \infty$. We work here with a Floquet operator of the explicit form

$$
\begin{aligned}
F= & \exp \left[-\mathrm{i} k_{z} \frac{J_{z}^{2}}{2 j+1}-\mathrm{i} p_{z} J_{z}\right] \exp \left[-\mathrm{i} p_{y} J_{y}\right] \\
& \times \exp \left[-\mathrm{i} k_{x} \frac{J_{x}^{2}}{2 j+1}-\mathrm{i} p_{x} J_{x}\right] .
\end{aligned}
$$

The dynamics consists of a sequence of linear rotations by angles $p_{i}$ alternating with torsions of strength $k_{i}$. We hold the $p_{i}$ fixed $\left(p_{x}=0.3, p_{y}=1.0, p_{z}=0.8\right)$ while varying the control parameter $k \equiv k_{z}=10 k_{x}$. Complete semiclassical spectra of this system throughout the full transition from integrable $(k=0)$ to well-developed chaotic behaviour $(k \approx 10)$ have been presented in [20]. The system has also been used to illustrate uniform semiclassical approximations for various kinds of bifurcations [5-7].

We concentrate on a particular configuration of periodthree orbits which suggests a treatment as a twofoldbroken torus. The configuration comes about in a sequence of three bifurcations: at $k=1.9715$ a pair of period-three satellites is born in close vicinity to a period-one orbit in the centre of a stability island. At $k=1.9753$ a periodtripling bifurcation takes place where the stable satellite collides with the central orbit. On the other side of the bifurcation the satellites form a simple broken torus around the centre. This sequence of bifurcations can be described by extended normal forms $[7,8]$, and the close neighbourhood of the two bifurcations is not exceptional (see e.g. [21]). As the control parameter $k$ is increased further the satellites move away from the centre. At $k=3.7856$ another pair of period-three satellites appears in a tangent bifurcation. For $k<5$ the four satellites form a configuration that resembles a twofold-broken torus. Figure 1 displays a phase space portrait for $k=4$, and Figure 2 shows steepest-descent contours in the complex $\varphi^{\prime}$-plane on both sides of the final bifurcation.

We evaluated $\operatorname{tr} F^{3}$ at $k=3.0$ with the quantum number $j$ ranging from 1 to 50. At the given value of the

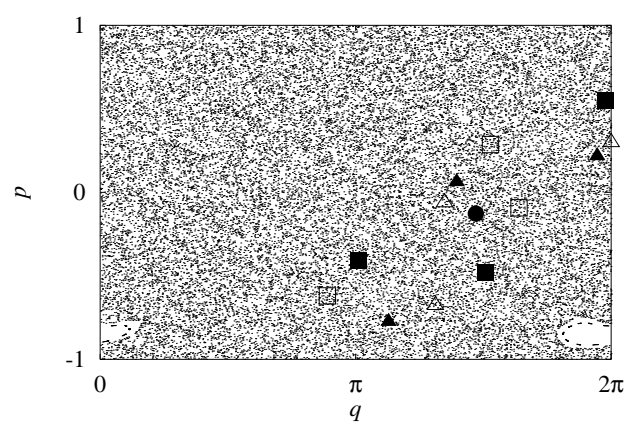

Fig. 1. Phase space portrait of the kicked top with $k=4.0$. The phase space is the unit sphere, parameterized here with the azimuthal angle $q$ and the $z$-component $p$ (in conventional spherical coordinates, $q=\phi$ and $p=\cos \theta$ ). The circle indicates the central period-one orbit, the other symbols indicate the positions of the four period-three satellites (all orbits are unstable).
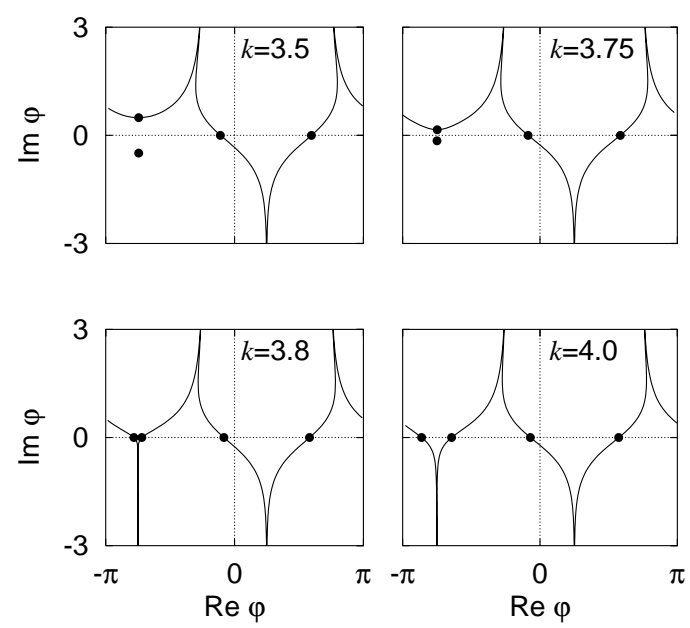

Fig. 2. Integration contours in the complex $\varphi^{\prime}$-plane on both sides of the tangent bifurcation in the kicked top at $k=3.7856$. For each $k$ the normal-form coefficients of the phase function $\Phi$ have been determined from the actions of the periodic orbits. The contours pass through the saddle points of the normal form (circles) and proceed in the direction of steepest descent (constant phase of $\Phi$ ).

control parameter only one of the pairs of satellites mentioned above has real coordinates, and its distance to the centre of the stability island is already quite large. The other two satellites are still ghosts, but their bifurcation is not far away. This leaves us with the choice between two semiclassical approximations: (i) we can group the two real satellites together with the central orbit and treat the ghosts separately, or (ii) we can group the four satellites as a twofold-broken torus and treat the central orbit separately. (In principle, we could complicate matters even more and group all the orbits together, but this is rather impractical and beyond the scope of the present work.) The semiclassical evaluation of the trace involves also five other orbits. The error of the two approximations is shown in Figure 3. The error of approximation (ii) 


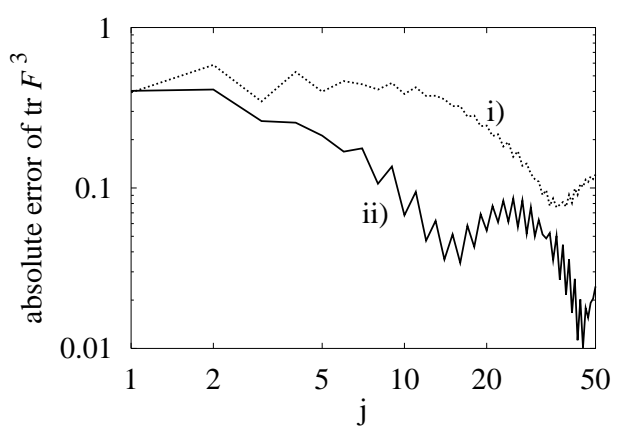

Fig. 3. Semiclassical error of $\operatorname{tr} F^{3}$ for the kicked top $(k=3.0)$ as a function of $j$ in the two approximations (i) and (ii) which are explained in the text.

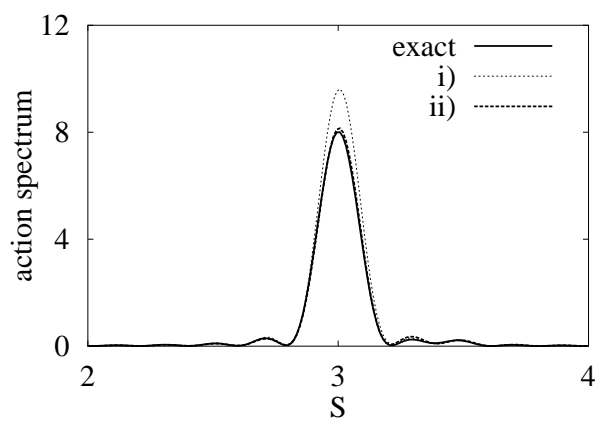

Fig. 4. Collective peak of the four satellites and the central orbit in the action spectrum $|T(S)|^{2}$ for $k=3.0$. The semiclassical approximations (i) and (ii) (see text) are compared to the result of an exact quantum-mechanical computation.

is about a factor 2-3 smaller than that of approximation (i). Although the accuracy gain is not dramatic, this result favours clearly a treatment of the satellites as a preformed twofold-broken torus.

A somewhat more demanding test is aided by "inverse$\hbar$ spectroscopy" $[9,22]$. We consider the discrete truncated Fourier analysis

$$
T(S)=\frac{1}{32} \sum_{j=1}^{32} \operatorname{tr} F^{3}(j) \exp \left[-\mathrm{i}\left(j+\frac{1}{2}\right) S\right]
$$

of the trace with respect to the quantum number $j$. The "action spectrum" $|T(S)|^{2}$ displays peaks at the actions of the periodic orbits that contribute to tr $F^{3}$. Since accidental action degeneracies do not occur in the present example, the quality of the semiclassical approximations (i) and (ii) can now be assessed directly, without interference of the remaining orbits. Figure 4 shows the collective peak of the four satellites and the central orbit. Approximation (ii) agrees almost perfectly with the exact result, while approximation (i) overestimates the peak-height distinctively.

Of course, the uniform approximation presented here is not restricted to the situation where two of the orbits are ghosts, but is valid for four real orbits on the broken torus as well. Indeed we find an improvement in the semiclassical accuracy of $\operatorname{tr} F^{3}$ over the full range $2.8<k<5$.

\section{Conclusion}

In this paper a uniform approximation for a broken rational torus consisting of four periodic orbits has been presented. The approximation was tested in a model system where the phase space is mixed, and the tori are grouped around (but not too close to) a central orbit. It can be expected that the approximation will be even more useful in studies of globally near-integrable systems.

This work was supported by the DFG (Sonderforschungsbereich 237) and the European Community (Program for the Training and Mobility of Researchers).

\section{References}

1. A.J. Lichtenberg, M.A. Lieberman, Regular and Chaotic Dynamics (Springer, New York, 1992).

2. A.M. Ozorio de Almeida, J.H. Hannay, J. Phys. A 20, 5873 (1987).

3. A.M. Ozorio de Almeida, Hamiltonian Systems: Chaos and Quantization (Cambridge University Press, Cambridge, 1988).

4. M. Sieber, J. Phys. A 29, 4715 (1996).

5. H. Schomerus, M. Sieber, J. Phys. A 30, 4537 (1997).

6. M. Sieber, H. Schomerus, J. Phys. A 31, 165 (1998).

7. H. Schomerus, Europhys. Lett. 38, 423 (1997).

8. H. Schomerus, J. Phys. A 31, 4167 (1998).

9. S. Tomsovic, M. Grinberg, D. Ullmo, Phys. Rev. Lett. 75, 4346 (1995); D. Ullmo, M. Grinberg, S. Tomsovic, Phys. Rev. E 54, 136 (1996).

10. J. Main, G. Wunner, Phys. Rev. Lett. 82, 3038 (1999).

11. M. Kuś, F. Haake, D. Delande, Phys. Rev. Lett. 71, 2167 (1993).

12. M.C. Gutzwiller, J. Math. Phys. 12, 343 (1971).

13. M.C. Gutzwiller, Chaos in Classical and Quantum Mechanics (Springer, New York, 1990).

14. M. Tabor, Physica D 6, 195 (1983).

15. G. Junker, H. Leschke, Physica D 56, 135 (1992).

16. D.A. Sadovskií, J.A. Shaw, J.B. Delos, Phys. Rev. Lett. 75, 2120 (1995).

17. D.A. Sadovskií, J.B. Delos, Phys. Rev. E 54, 2033 (1996).

18. J. Main, G. Wunner, Phys. Rev. A 55, 1743 (1997).

19. F. Haake, Quantum Signatures of Chaos (Springer, Berlin, 1990); F. Haake, M. Kuś, R. Scharf, Z. Phys. B 65, 381 (1987); M. Kuś, F. Haake, B. Eckhardt, Z. Phys. B 92, 221 (1993); P. Braun, P. Gerwinski, F. Haake, H. Schomerus, Z. Phys. B 100, 115 (1996).

20. H. Schomerus, F. Haake, Phys. Rev. Lett. 79, 1022 (1997).

21. J.-M. Mao, J.B. Delos, Phys. Rev. A 45, 1746 (1992).

22. D. Wintgen, Phys. Rev. Lett. 58, 1589 (1987); U. Eichmann, K. Richter, D. Wintgen, W. Sander, Phys. Rev. Lett. 61, 2438 (1988). 